\title{
RASCAL FINITE SETS
}

\author{
PIER LUIGI PAPINI
}

\begin{abstract}
In this paper we consider finite sets in a normed, infinite dimensional space. First, we study the following problem: given a finite set $F$, does there exist a sphere containing $F$ on its surface? We indicate some results and we collect some examples concerning this problem, also for sets of small cardinality. Then we give an example of a three-point set, in a Hilbert space, without incenter.
\end{abstract}

\section{Introduction}

We shall denote by $(X,\|\|$.$) a normed space over the real field R ; F$ will always indicate a finite subset of $X$. Also, we assume that $X$ is infinite-dimensional, though several results can be stated for spaces having a finite dimension "large enough" with respect to $\# F$, the number of elements of $F$.

For $x \in X$ and $r>0$, we denote by $U(x, r)$ the set $\{y \in X ;\|x-y\|=r\}$.

As usual, we denote by $l_{p}, 1 \leq p<\infty$, the space of all sequences $x=\left(x_{1}, \ldots, x_{n}, \ldots\right)$ such that $\sum_{i=1}^{\infty}\left|x_{i}\right|^{p}$ is summable, with the norm $\|x\|=\left(\sum_{i=1}^{\infty}\left|x_{i}\right|^{p}\right)^{1 / p}$.

A subset $A$ of $X$ is said to be equilateral if $\|x-y\|$ is constant for all pairs $x, y$ in $A$.

We say that $F$ lies on a sphere if there exist $x \in X$ and $r>0$ such that $F \subset U(x, r)$.

Clearly, if $F$ contains two points then it always lies on a sphere.

We shall consider the following property, which was introduced and studied in [3] for a normed space $X$ :

$(P) \quad$ any finite subset of $X$ lies on a sphere.

Here we deal with spaces having or lacking property $(P)$, and we indicate some related results: only an infinite-dimensional space can have property $(P)$. Other connected results have been indicated in [4] and in [11].

\section{Triplets Versus Quadruplets}

We start with a few simple remarks.

It is clear that if $X$ is strictly convex and $F$ contains (at least) three collinear points, then $F$ cannot lie on a sphere: in fact, this property characterizes strictly convex spaces.

Received June 20, 2003; revised October 17, 2003.

2000 Mathematics Subject Classification. 46B99, 52C35, 90B85.

Key words and phrases. sets lying on a sphere, equilateral sets, incenter. 
If the norm of $X$ is Hilbertian, then it is simple to see that every finite, equilateral set lies on a sphere (it is enough to consider the centroid of the set).

If $\# F=3$ and $F$ is equilateral, then we can add a point to $F$ so that the new set is still equilateral (see [12]): this implies that, in any space, every three-point equilateral set lies on a sphere. Still in [12] an example of a maximal, equilateral set containing 4 points was given.

An example of a Banach space containing an equilateral set $F$ of 4 points, not lying on a sphere, was given in [2], Example 6.1.

In many Banach spaces not every three-point, non collinear set, lies on a sphere. More precisely, the following is true (see [10], Proposition 41):

A Minkowski plane is smooth iff every noncollinear triplet lies on a sphere.

Compare with Example 3 below; see also the discussion at the beginning of Section 3.

We give now some examples of triplets or quadruplets not lying on a sphere; clearly, all the spaces involved lack property $(P)$.

Example 1. Let $X=l_{1}$. Let $F=\left\{e_{1},-e_{1}, \alpha e_{2}\right\}, 0<\alpha<1, e_{i}$ denoting the $i$-th element of the natural basis; note that $F$ is not collinear. Assume that $F \subset U(c, \rho)$ for some $c=\left(c_{1}, \ldots, c_{n}, \ldots\right) \in X$ and some $\rho>0$. From $\left\|c-e_{1}\right\|=\left\|c+e_{1}\right\|$ we obtain $c_{1}=0$; moreover, from ||$c-e_{1}||=|| c-\alpha e_{2}||$ we obtain $1+\left|c_{2}\right|=\left|c_{2}-\alpha\right|$ : this is a contradiction, proving that $F$ cannot lie on a sphere.

If we consider a set like $F$ in $l_{p}, 1<p<\infty$, then $1+\left|c_{2}\right|^{p}=\left|c_{2}-\alpha\right|^{p}$ gives a contradiction only for $\alpha=0$ (note that in this case the three points are collinear).

Example 2. Let $X=c_{o} ; x=\left(1, \frac{1}{2}, \frac{1}{3}, \frac{1}{4}, \frac{1}{5}, \ldots\right) ; y=\left(-\frac{1}{2},-1,-\frac{1}{3},-\frac{1}{4},-\frac{1}{5}, \ldots\right) ; F=$ $\{x, y, \theta\}$ ( $F$ is not collinear). Assume that $F \subset U(c, r)$ for some $c=\left(c_{1}, \ldots, c_{n}, \ldots\right) \in X$ and some $r>0$ : then $\|c\|=\|c-\theta\|=r$; if $\left|c_{n}\right|=r$ for some $n \geq 3$, then $\max \left\{\left|c_{n}-x_{n}\right|\right.$, $\left.\left|c_{n}-y_{n}\right| ; n \geq 3\right\}=r+\frac{1}{n}$, so either $\|c-x\|>r$, or $\|c-y\|>r$. If $\|c-\theta\|=\left|c_{1}\right|=r$, then either $c_{1}<0$, so $\| c-x|| \geq\left|c_{1}-x_{1}\right|=1+r>r$, or $c_{1}>0$, so $\|c-y\| \geq\left|c_{1}-y_{1}\right|=\frac{1}{2}+r>r$; similar implications, if $\| c-\theta||=\left|c_{2}\right|=r$. This contradiction proves that $F$ does not lie on a sphere.

For $X=c$ (the space of all convergent sequences), or $X=l_{\infty}$, a similar example can be given, by taking $F=\{x, y, \theta\}$ with $x=(1,-2,1,1,1, \ldots) ; y=(-2,1,-1,-1,-1, \ldots)$. In this case, also by assuming $\|c\|=r=\varlimsup_{\lim _{n \rightarrow \infty}}\left|c_{n}\right|$ we get a contradiction.

Example 3. Let $X=l_{p}, 1 \leq p<\infty$. Consider the following subset of $X$ : $F=$ $\left\{e_{1},-e_{1}, \alpha e_{2},-\alpha e_{2}\right\}$ where $0<\alpha<1$ and $e_{1}, e_{2}$ are the first two elements of the natural basis. Note that no triplet in $F$ is collinear.

Assume that $F \subset U(c, r)$ for some $c=\left(c_{1}, c_{2}, \ldots\right) \in X$ and some $r>0:\left\|c-e_{1}\right\|=$ $\left\|c+e_{1}\right\|$ implies $c_{1}=0$; also, $\left\|c-\alpha e_{2}\right\|^{p}=\left\|c+\alpha e_{2}\right\|^{p}$ implies $c_{2}=0$. Moreover, $\left\|c-e_{1}\right\|=\| c-\alpha e_{2}||$ implies $1+\sum_{n \geq 3}\left|c_{n}\right|^{p}=\alpha^{p}+\sum_{n \geq 3}\left|c_{n}\right|^{p}$, a contradiction proving that $F$ does not lie on a sphere. 


\section{Some Positive Results}

In [3] two examples of spaces where finite sets always lie on a sphere have been given: one is a smooth (not complete) normed space; the other one is a complete (but not smooth) space.

Here we indicate an example of a simple normed space with property $(P)$.

Example 4. Let $X$ be the space of all real sequences $x=\left(x_{1}, \ldots, x_{n}, \ldots\right)$ with a finite number of non null components, with the norm:

$\|x\|=\max \left\{\left|x_{i}\right|, 1 \leq i<\infty\right\}$.

Assume that $a_{1}, \ldots, a_{n}$ are the elements of $F$, with components $\left(a_{i}\right)_{j}, 1 \leq i \leq n$ and $j \in N$. Let $\alpha=\max _{1 \leq i \leq n}\left\|a_{i}\right\|$, and $n_{o}$ such that $\left(a_{i}\right)_{j}=0$ for $j \geq n_{o}$ and $i=1, \ldots, n$.

Take $x=\alpha e_{n_{o}}\left(e_{n_{o}}\right.$ being the $n_{o}$-th element of the natural basis); then $F \subset U(x, \alpha)$.

Remark 1. If we complete the above space, then property $(P)$ fails (see Example $2)$.

A "positive" result is the following.

Proposition 1. Any space $X$ can be embedded as a norm-one complemented hyperplane of a space $Y$ so that for every finite subset $F$ of $X$, the subset in $Y$ corresponding in a natural way to $F$ lies on a sphere.

Proof. Given $X$, let $Y=(R \oplus X)_{\infty}$. If $F=\left\{x_{1}, \ldots, x_{n}\right\} \subset X$ and $\max _{1 \leq i \leq n}\left\|x_{i}\right\|=$ $\alpha$, let $x_{i}^{\prime}=\left(0, x_{i}\right)(1 \leq i \leq n)$. Then $F^{\prime}=\left\{x_{1}^{\prime}, \ldots, x_{n}^{\prime}\right\} \subset U(c, \alpha)$ where $c=(\alpha, 0)$.

Remark 2. The above Proposition does not say that $(R \oplus X)_{\infty}$ satisfies $(P)$ (see $[3$, Example 1]).

If $X$ is smooth, then the following is true (see [3], Theorem 2).

$\left(\mathrm{P}^{\prime}\right)$ given $x_{1}, \ldots, x_{n}$ in $X$ and $\varepsilon>0$, we can find for $i=1, \ldots, n$ points $x_{i}^{\prime}$ such that $\left\|x_{i}-x_{i}^{\prime}\right\| \leq \varepsilon$ and $x_{1}^{\prime}, \ldots, x_{n}^{\prime}$ lie on a sphere.

This result relies on "Vlasov's property" (see [13], p.35). More precisely, spaces with a strictly convex dual are characterized by the following property: the union of an increasing, unbounded sequence of open balls is either an open halfspace, or the whole space.

Since we deal with finite sets (generating finite-dimensional spaces), we recall that when $\operatorname{dim}(X)<\infty, X$ is smooth if and only if the dual is strictly convex.

The above result holds also in $c_{o}$; the proof given below simply relies on the fact that the space considered in Example 4 is dense in $c_{o}$.

Proposition 2. The space $c_{o}$ satisfies property $\left(P^{\prime}\right)$.

Proof. Let $X=c_{o}$; given $x_{1}, \ldots, x_{n}$, set $\max _{1 \leq i \leq n}\left\|x_{i}\right\|=\alpha$. Take a positive $\varepsilon<\alpha$, then find $n_{o} \in N$ such that $\left(x_{i}\right)_{j} \leq \varepsilon$ for $j \geq n_{o}$. Set $x_{i}^{\prime}=\left(\left(x_{i}\right)_{1}, \ldots,\left(x_{i}\right)_{n_{o}-1}, 0, \ldots, 0, \ldots\right)$ $(1 \leq i \leq n)$; then we have: $\left\|x_{i}-x_{i}^{\prime}\right\| \leq \varepsilon$. Moreover $\left\{x_{1}^{\prime}, \ldots, x_{n}^{\prime}\right\} \subset U(x, \alpha)$ where $x=\alpha e_{n_{o}}$. 
Remark 3. We note that property $(P)$ lifts for example to finite $l_{p}$ products of spaces, $1 \leq p \leq \infty$. The same is true when the product is performed by using a monotone norm, or also more general types of norms (see [7], [9]).

\section{Concerning (in)Stability; Nearly Collinear Sets and Big Spheres}

Next examples show that, also when the norm is Euclidean, small perturbations of a set $F$ lying on a sphere can imply big changes concerning the center or the size of the sphere containing it; the finite sets we consider lie on a sphere.

Example 5. In this example, we indicate two sets "near" to each other, while the spheres containing them (which have the same radius) have "distant" centers.

Let $X$ be the Euclidean plane; we consider two sets which are not collinear (but are not far from being collinear). Take $F=\left\{(0,0) ;\left(\varepsilon, \sqrt{1-\varepsilon^{2}}-1\right) ;\left(-\varepsilon, \sqrt{1-\varepsilon^{2}}-1\right)\right\}$ $(\varepsilon<1)$ : then the (unique) sphere containing $F$ is centered at $c=(0,-1)$ and has radius $r=1$.

Now take $F^{\prime}=\left\{(0,0) ;\left(\varepsilon, 1-\sqrt{1-\varepsilon^{2}}\right) ;\left(-\varepsilon, 1-\sqrt{1-\varepsilon^{2}}\right)\right\}$ : then the (unique) sphere containing $F$ is centered at $c^{\prime}=(0,1)$ and has radius $r=1$. The Hausdorff distance between $F$ and $F^{\prime}$ is $2 \sqrt{1-\varepsilon^{2}}$, which is near to 0 for $\varepsilon$ small; but $\left\|c-c^{\prime}\right\|=2$ (so their centers are not near).

Example 6. In this example, we have sets "near" to each other, while the radii of the spheres containing them are "very different".

Let $X$ be the Euclidean plane. Take $G=\left\{(0,0) ;\left(\sqrt{2 \varepsilon-\varepsilon^{2}}, \varepsilon\right) ;\left(-\sqrt{2 \varepsilon-\varepsilon^{2}}, \varepsilon\right)\right\}$ with $\varepsilon<1$ : then the (unique) sphere containing $F$ is centered at $c=(0,1)$ and has radius $r=1$.

Now take $G^{\prime}=\{(0,0) ;(\varepsilon, \varepsilon) ;(-\varepsilon, \varepsilon)\}$ : then the (unique) sphere containing $F$ is centered at $c^{\prime}=(0, \varepsilon)$ and has radius $r^{\prime}=\varepsilon$. Therefore the Hausdorff distance between $G$ and $G^{\prime}$ is $\sqrt{2 \varepsilon-\varepsilon^{2}}-\varepsilon$, while the difference $r-r^{\prime}$ is $1-\varepsilon$.

We consider now collinear sets.

We observe first that the radius $r$ of a sphere containing a set $F=\left\{x_{1}, \ldots, x_{n}, \ldots\right\}$ must be not smaller than the (Chebyshev) radius of $F$; i.e.,

$r \geq \inf _{x \in X} \sup _{1 \leq i \leq n}|| x-x_{i} \|$

If $X$ is strictly convex, then a collinear triplet cannot lie on a sphere.

Also: if $X$ is uniformly convex, then we can estimate -by using the modulus of convexityhow far a set $F$ consisting of three collinear points is, from lying on a sphere of radius $r$. More precisely, if we consider a small perturbation of a collinear set $F$, a sphere of big radius is necessary to contain $F$ : spheres can be "near to" collinear triplets only if their radii are "big enough", depending on the roundness of the space.

For sake of simplicity, we indicate an estimate for spaces whose norm derives from an inner product. The extension to uniformly convex spaces would be not too difficult. 
Let $X$ be a Hilbert space; recall that $X$ is uniformly convex, with modulus of convexity

$$
\delta(\varepsilon)=1-\sqrt{1-\frac{\varepsilon^{2}}{4}} .
$$

This means that given $x, y$ in $X$,

if $\|x\| \leq r ;\|y\| \leq r ;\|x-y\| \geq \varepsilon$, then $\left\|\frac{x+y}{2}\right\| \leq r\left(1-\delta\left(\frac{\varepsilon}{r}\right)\right)$.

Proposition 3. Let $X$ be an inner product space; assume that $F$ is a three-point set, containing two points and the middle point of the segment, of length $2 \alpha$, they determine. Then in order to obtain a set lying on a sphere of radius $\rho$, it is enough to move the points of $\frac{\alpha^{2}}{4 \rho}$; it is necessary to move at least one of them of $\frac{2 \rho+\alpha-2 \sqrt{\rho^{2}-\alpha^{2}+\alpha \rho}}{5}$.

Proof. Let $F=\{-x, \theta, x\}$ and $\|x\|=\alpha$. Set:

$E(-x, x)=\{y \in X ;\|y+x\|=\|y-x\|\}$.

If $X$ is an inner product space, then $E(-x, x)$ is nothing else than the hyperplane (through $\theta$ ) orthogonal to $x$.

We want to give an upper estimate.

Take on $E(-x, x)$ a point $c \neq \theta$, then consider $U(c, \rho)$; also, set $\|c-x\|=d(>\alpha)$. In order to have $-x, \theta, x$ near to $U(c, \rho)$, we can ask, for example, that:

$$
\rho-\|c\|=\|c-x\|-\rho .
$$

We have $\|x-(-x)\|=2 \alpha$, and then, by using (1), $\|c\|=\left\|c-\left(\frac{x+(-x)}{2}\right)\right\|=d\left(1-\delta\left(\frac{2 \alpha}{d}\right)\right)$. So (2) becomes $d-\rho=\rho-d\left(1-\delta\left(\frac{2 \alpha}{d}\right)\right)$, or $\rho=d-\frac{d}{2} \delta\left(\frac{2 \alpha}{d}\right)=d-\frac{d}{2}\left(1-\sqrt{1-\frac{\alpha^{2}}{d^{2}}}\right)$.

This shows that we obtain a triplet lying on a sphere, by moving the elements of $F$ of

$$
d-\rho=\frac{d}{2}-\sqrt{\frac{d^{2}-\alpha^{2}}{4}}=\frac{1}{2}\left(d-\sqrt{d^{2}-\alpha^{2}}\right) .
$$

Of course, the last term gives a decreasing function of $d$, which goes to 0 as $d \rightarrow \infty$.

This result can be formulated in the following way ( $\operatorname{set} d-\rho=\varepsilon$ ).

If we want to change the three points of the collinear set $F$ as given, so that the new set lies on a sphere of radius $\rho$, it is enough to move them of

$$
\varepsilon=\frac{1}{2}\left(\rho+\varepsilon-\sqrt{(\rho+\varepsilon)^{2}-\alpha^{2}}\right)(\rho<\varepsilon) \Longleftrightarrow \rho-\varepsilon=\sqrt{(\rho+\varepsilon)^{2}-\alpha^{2}} ;
$$

but according to (2), $2 \rho-d>0$, thus $\rho-\varepsilon>0$; so the last equality is equivalent to $\varepsilon=\frac{\alpha^{2}}{4 \rho}$.

Now we see the problem from the other side, in order to obtain a lower estimate. Take again $F$ as before. Assume that we can find $F^{\prime}=\{u, v, z\}$, lying on the sphere $U(c, \rho)$, and such that:

$$
\|u+x\| \leq \varepsilon ; \quad\|v-\theta\| \leq \varepsilon ; \quad\|z-x\| \leq \varepsilon ; \quad(\alpha>\varepsilon) .
$$


Set $u=-x+x^{\prime} ; z=x+x^{\prime \prime}$; we have:

$\left\|\frac{u+z}{2}\right\|=\left\|\frac{x^{\prime}+x^{\prime \prime}}{2}\right\| \leq \varepsilon ; \quad\left\|c-\frac{u+z}{2}\right\| \geq\|c-v\|-\|v-\theta\|-\left\|\theta-\frac{u+z}{2}\right\| \geq \rho-2 \varepsilon$.

So, if we assume $\varepsilon<\frac{\rho}{2}$, the ball of radius $\rho$ centered at $c$ contains the segment $[u, z]$, of length $\geq 2 \alpha-2 \varepsilon$, and the middle point has a distance from $c$ not smaller than $\rho-2 \varepsilon$. In other terms, the unit ball of $X$ contains a segment of length at least $\frac{2 \alpha-2 \varepsilon}{\rho}$, and the distance of its middle point from $c$ is $\geq 1-\frac{2 \varepsilon}{\rho}$. According to (1), this implies:

$$
1-\delta\left(\frac{2 \alpha-2 \varepsilon}{\rho}\right) \geq 1-\frac{2 \varepsilon}{\rho}, \quad \text { or } \quad \delta\left(\frac{2 \alpha-2 \varepsilon}{\rho}\right)=1-\sqrt{1-\frac{1}{4}\left(\frac{2 \alpha-2 \varepsilon}{\rho}\right)^{2}} \leq \frac{2 \varepsilon}{\rho},
$$

$\mathrm{SO}$

$\sqrt{1-\left(\frac{\alpha-\varepsilon}{\rho}\right)^{2}} \geq 1-\frac{2 \varepsilon}{\rho} \Longleftrightarrow \rho^{2}-(\alpha-\varepsilon)^{2} \geq(\rho-2 \varepsilon)^{2} \Longleftrightarrow 5 \varepsilon^{2}-2 \varepsilon(2 \rho+\alpha)+\alpha^{2} \leq 0$.

Therefore, we obtain:

$$
\varepsilon \geq \frac{2 \rho+\alpha-2 \sqrt{\rho^{2}-\alpha^{2}+\alpha \rho}}{5} .
$$

This completes the proof.

\section{A Three-Point Set Without Incenter}

Recall that the following problem, related to property $\left(P^{\prime}\right)$, is an important one for applications (see [1], [5]): given a finite set $F$, find a "small" annulus containing $F$. The discussion in the previous section is related also to this problem. In fact, if we say that the size of the annulus is connected with the difference between those of the inner and the outer circle, we have seen that "size 0" is not usual, but "small size" is possible if the balls are big enough.

Another important problem, often considered in location theory (see for example [6]) is the following one:

Given a set $A$, find a point $x \in Y, Y$ given, such that

$$
\inf _{a \in A}\|x-a\|=\sup _{y \in Y} \inf _{a \in A}\|y-a\| .
$$

When such a point $x$ exists, it is called an incenter of $A$.

If $A$ is assumed to be bounded, then the problem makes sense only if $Y$ is bounded (otherwise $\inf _{a \in A}\|x-a\|$ goes to infinity for $x$ going far from $A$ ).

Here we shall assume that we have a finite set $F=\left\{a_{1}, \ldots, a_{n}\right\}$, and that $Y$ is closed and bounded; moreover, we assume that the underlying space $X$ is a Banach space (without completeness of $X$, we can hardly hope for existence of solutions). These 
assumptions are usual done in applications, where in general $X$ is finite-dimensional (so the problem is also called a "maxmin" problem).

In case we take $Y=c o(F)$, then there always exists some $c$ such that

$$
\min _{a \in F}\|c-a\|=\max _{x \in Y} \min _{a \in F}\|x-a\|
$$

In fact, $c o(F)$ has finite affine dimension, so the problem becomes finite-dimensional and then compactness implies existence of solutions.

The above problem $(*)$ was studied from a general point of view, for $A$ a convex set. The existence of solutions is then related to the structure of the underlying Banach space: see for example [8].

We indicate now, for $X$ a Hilbert space, an example of a three-point set without incenter.

Example 7. Let $X=l_{2}$, and $F=\left\{a_{1}, a_{2}, a_{3}\right\}$ where:

$$
\begin{aligned}
& a_{1}=(0,1,0,0, \ldots) \\
& a_{2}=\left(\frac{\sqrt{3}}{2},-\frac{1}{2}, 0,0, \ldots\right) \\
& a_{3}=\left(-\frac{\sqrt{3}}{2},-\frac{1}{2}, 0,0, \ldots\right) .
\end{aligned}
$$

Let $Y=\left\{x \in X ;\left|x_{1}\right| \leq \frac{\sqrt{3}}{2} ;\left|x_{n}\right| \leq 2-\frac{1}{n-1}\right.$ for $n \geq 2 ; \sum_{n=1}^{\infty}\left|x_{n}\right| \leq 2$, so $\left.\|x\| \leq 2\right\}$.

Given $x \in Y$, let $x^{\prime}$ be the orthogonal projection of $x$ on the two-dimensional subspace generated by $e_{1}$ and $e_{2}$; also, denote by $Z$ the projection of $Y$ onto this subspace ( $Z$ is a rectangle).

For every $z \in Z$, we have the estimate $\min _{1 \leq j \leq 3}|| z-a_{j} \| \leq 1$. Therefore, if $x \in Y$, then $\min _{1 \leq j \leq 3}\left\|x-a_{j}\right\|^{2}=\min _{1 \leq j \leq 3}\left(\left\|x-x^{\prime}\right\|^{2}+\left\|x^{\prime}-a_{j}\right\|^{2}\right)$. Since $x^{\prime} \in Z, \min _{1 \leq j \leq 3}\left\|x-a_{j}\right\| \leq$ $\sqrt{2^{2}+\min _{1 \leq j \leq 3}\left\|x^{\prime}-a_{j}\right\|^{2}} \leq \sqrt{5}$.

Now take, for $n \geq 3, c_{n}=\left(0,0, \ldots, 2-\frac{1}{n}, 0, \ldots\right)$, only the $(n+1)-t h$ coordinate being $\neq 0$. We have:

$$
\left\|c_{n}\right\| \leq 2 ; \quad c_{n} \in Y
$$

also, for $j=1,2,3\left(\left\|a_{j}\right\|=1\right)$ :

$$
\left\|c_{n}-a_{j}\right\|=\sqrt{1+\left(2-\frac{1}{n}\right)^{2}}=\sqrt{5+\frac{1}{n^{2}}-\frac{4}{n}} \underset{n \rightarrow \infty}{\longrightarrow} \sqrt{5}
$$

Therefore $\sup _{x \in Y} \min _{a \in A}\|x-a\| \geq \sqrt{5}$.

But we cannot have, for $x \in Y, \min _{a \in A}\|x-a\| \geq \sqrt{5}$. In fact, this would imply $\left\|x-a_{j}\right\|=1$ for all $j$, so $x_{1}=x_{2}=0 ; 2=\sqrt{\sum_{n=3}^{\infty}\left|x_{n}\right|^{2}} \leq \sum_{n=3}^{\infty}\left|x_{n}\right| \leq 2$, which is impossible since there must be more than one component of $x$ different from 0 . Therefore $F$ has no incenter. 


\section{Acknowledgements}

The author wishes to thank the referee for some useful comments and remarks.

The research was partially supported by the Italian National Research Group G.N.A.M.P.A.

\section{References}

[1] P. K. Agarwal, B. Aronov, S. Har-Peled and M. Sharir, Approximation algorithms for minimum-width annuli and shells, Discrete Comput. Geom. 24 (2000), 687-705.

[2] M. Baronti, E. Casini and P. L. Papini, Equilateral sets and their central points, Rend. Mat. 13 (1993), 133-148.

[3] Á.P. Bosznay, A property of spheres in normed linear spaces, Ann. Univ. Sci. Budapest. Eötvös Sect. Math. 29 (1986), 183-188 (1987).

[4] P. Brass, On equilateral simplices in normed linear spaces, Beiträge Alg. Geom. 40 (1999), 303-307.

[5] Z. Drezner, S. Steiner, and G. O. Wesolowsky, On the circle closest to a set of points, Comput. Oper. Res. 29 (2002), 637-650.

[6] E. Erkut and S. Neuman, Analytical models for locating undesirable facilities, Eur. J. Oper. Res. 40 (1989), 275-291.

[7] C. R. Johnson and P. Nylen, Monotonicity properties of norms, Linear Algebra Appl. 148 (1991), 43-58.

[8] V. Klee, E. Maluta and C. Zanco, Inspheres and inner products, Israel J. Math. 55 (1986), $1-14$.

[9] C.-K. Li and N.-K. Tsing, Norms on cartesian product of linear spaces, Tamkang J. Math. 21 (1990), 35-39.

[10] H. Martini, K. J. Swanepoel, G. Weiss, The geometry of Minkowski spaces - A survey. Part I, Exposition. Math. 19 (2001), 97-142.

[11] Papini, P. L., Equilateral, diametral, centered sets and subsets of spheres, Extracta Math. 19(2004), 261-267.

[12] Petty, C. M., Equilateral sets in Minkowski spaces, Proc. Amer. Math. Soc. 29 (1971), 369-374.

[13] L. P. Vlasov, Approximative properties of sets in normed linear spaces, Russian Math. Surv. 28 (1973), 1-66.

Pier Luigi Papini, Dipartimento di Matematica, Piazza Porta S. Donato, 5, 40126 Bologna, Italy.

E-mail: papini@dm.unibo.it 